\title{
CONTEXTUALIZING A PROFESSIONAL SOCIAL NETWORK FOR HEALTHCARE: EXPERIENCES FROM AN ACTION DESIGN RESEARCH STUDY
}

Tobias Mettler, University of Lausanne, Swiss Graduate School of Public Administration, Rue de la Mouline 28,CH-1022 Chavannes-près-Renens, Switzerland, tobias.mettler@unil.ch

Citation: Mettler T. Contextualizing a professional social network for health care: Experiences from an action design research study. Info Systems J. 2017; https://doi.org/10.1111/isj.12154

\begin{abstract}
.
Professional social networks (PSN) are online environments where practitioners can present themselves, get in contact and socialize with coworkers, share and discuss ideas, or exchange business-related knowledge. Despite the fact that collaboration and information sharing are becoming more relevant for delivering high-quality services, PSN are not yet widely adopted in complex domains such as healthcare. While most of the literature is still focusing on the exposition of the unbound potential of PSN, this paper seeks to clarify the question of how to capture and manage the professional identity of an industry such that a PSN can be purposefully anchored in the working context. Following an Action Design Research (ADR) approach, we describe practical design propositions and possible tensions along the contextualization of a PSN, which was specifically catered for improving interprofessional and inter-organizational collaboration in and between hospitals. We identify several implications for future research. In particular, we explain intended and unintended uses of PSN in hospitals and provide metaphors for explaining possible alternative understandings of domain engineering.
\end{abstract}

\section{Keywords.}

Action design research, domain engineering, enterprise social systems, healthcare, professional social networks. 


\section{INTRODUCTION}

Long before the rise of social media, offline professional social networks (PSN) have been a means for sustaining shared norms, solving asymmetries of information, stimulating the exchange and dissemination of new insights, and successfully undertaking collective action (Epstein, 1998). As more and more of our private and business life is moving to the digital world, this collective action nowadays often takes place in online environments where business professionals can present themselves, get in contact and socialize with coworkers, share and discuss ideas, find new job prospects, or exchange business-related information and knowledge (Musiał and Kazienko, 2013).

Like other social media applications such as blogs/micro-blogs (Zhang et al., 2010; Zhao and Rosson, 2009), wikis (Stocker et al., 2012), or instant-messaging (Cameron and Webster, 2005) a key characteristic of online PSN is to make behaviors, preferences, relationships, and knowledge unrestrictedly perceptible to others (Cross et al., 2003; Leonardi, 2014). According to Treem and Leonardi (2013), this increased visibility has the potential to present information communally, which means contributions can be easily located and viewed by other coworkers, as well as even generate meta-knowledge that is not available in any traditional knowledge repositories or elsewhere. In addition, posts, comments, status updates, votes, revisions, and other forms of information sharing frequently afford improved transparency of ongoing or planned organizational activities (Zhao and Rosson, 2009) and is therefore invaluable for the corporate setting (Argyris and Monu, 2015). Particularly in the healthcare industry, where collaboration and knowledge exchange are crucial for delivering high-quality services (Cnossen, 2014; Merrick et al., 2009), PSN may unfold new opportunities in the discovery of expertise, facilitation of cross-boundary collaboration, preservation of the institutional memory, and harness of distributed knowledge of health workers and patients (Miller et al. 2011). Some studies even predict that PSN may provoke a decline of inter-organizational and inter-professional boundaries and thus pave the way for new patterns how healthcare professionals may communicate with each other in the future (Jones and Thomas, 2007; Kamel Boulos and Wheeler, 2007; Reagans et al., 2004).

In order to achieve these promised benefits, unlike other social media applications that frequently concentrate on a specific facet of social interaction such as sharing (e.g. Pinterest, YouTube), conversations (e.g. Skype, WhatsApp), presence (e.g. Foursquare, Twitter), or relationships (e.g. Facebook, Google+) (Kietzmann et al. 2011), PSN need to 
possess additional qualities for supporting the development of mutual interests and common identity of a profession and for reducing existing professional barriers which hinder the exchange and dissemination of best practices and novel ideas. Accordingly, contextualization —or in other words, the adaptation to local, linguistic, historical, political, professional, and social conditions- has been identified as key success factor such that ITbased solutions in general, and PSN in particular, become valuable, meaningful, and acceptable for healthcare professionals (Chiasson and Davidson, 2004; Fichman et al., 2011; Haddad et al., 2014).

Although prior literature has largely focused on explaining the unbound potential of PSN for healthcare (Camlek 2015; Luo and Smith, 2010), little emphasis has been given to the question of how to capture and manage the professional identity of an occupational group (or even the entire industry) and how to use these insights for contextualizing a generic PSN platform in order that it is purposefully anchored in the work environment. Following this thread, and because existing approaches to domain engineering tend to be mechanistic and often lack a pluralistic process to identity and community building, it is the aim of this paper to discuss both specific design propositions and possible tensions along the contextualization of a PSN specifically catered for inter-professional and interorganizational exchange in healthcare. In doing so, we contribute to the ongoing discussions on the important role of the "industry" and "context" in information systems design (Bricon-Souf and Newman, 2007; Chiasson and Davidson, 2005; Lieberman and Selker, 2000) by facilitating theoretical narratives for describing alternative conceptions of domain engineering and providing practical insights regarding conceivable stumbling blocks when adapting PSN to the healthcare context.

In what follows, we review the relevant work on PSN in healthcare, analyze existing solutions for this domain, and clarify the research gap. After briefly explaining our methodological approach, we then describe the major solution components of our domainspecific PSN. This is followed by a reflection on the practical and theoretical learning outcomes in form of design principles and metaphors, respectively. We conclude with a discussion concerning the validity and limitations of our approach and give recommendations for future research. 


\section{BACKGROUND}

\section{Current use of PSN in healthcare}

Healthcare has been very receptive to technological inventions since its beginnings (Reiser, 1981). Most notably, adoption of new technologies has advanced rapidly in areas where it facilitates the care and cure of diseases. Despite the growing complexity of medical treatments and the increased pressure to perform more efficiently and cost effectively (Cleven et al., 2014), PSN have not yet received great attention as possible solution for improving collaborative medical decision-making and knowledge exchange in medical practice.

A recent study conducted by AMN Healthcare (2013) found that among the 85 percent of the U.S. health workers who admitted to use social network-like platforms, 55 percent say they use it primarily for personal purposes, 19 percent use it equally for professional and personal purposes and only 11 percent use it primarily for professional purposes, however, referring mainly to job seeking or professional networking. The little professional use of social networks is also supported by empirical findings by Alkhateeb et al. (2011). The aforementioned study also discovered significant differences in usage behavior of the varying professional groups. While registered nurses and medical students range at the top of the usage scale, physicians seem to be the least likely professional group to use social networks. The reasons for that seem to be inherent in the dichotomy of the nature of social technology and established patterns of physician decision-making: Bruno et al. (2011) argue that social technology is built according the ideas of egalitarianism, weak ties, coproduction, and voluntary sharing with the intention of 'encouraging a maximum of contributors and of getting the best solution by fusing a high number of contributions.' McAfee (2009) states that the key asset of social technologies is to facilitate informal, less structured, more spontaneous knowledge exchange.

In contrast to that, traditional physician decision-making frequently is based on discrete, disciplinary expert opinion (Lipman, 2000) or well researched and structured information, mainly originating from randomized clinical trials and meta-analyses (Sniderman et al., 2013). The notion of discussing treatment decisions at the same eye level with patients or with younger colleagues and based on fuzzy information requires a major paradigm shift in medical education (Anderson and Funnell, 2005). According to Wiener et al. (2012), using social networks also bears exceptional risks for physicians. Following Cain (2008) and Jain 
(2009), physicians never know how a conversation will shape and turn over time and who will read it in the end. Therefore physicians expressed great concerns related to privacy and confidentiality issues when being asked about using social networks (Lefebvre and Bornkessel, 2013; Mansfield et al., 2011; White et al., 2013).

In part as response to these concerns, the American Medical Association (2010) framed regulations for the appropriate use of social technologies, explicitly encouraging health workers to safeguard personal information, maintaining appropriate boundaries and confidentiality and keeping in mind that online content can negatively affect the reputation and career. Furthermore, the British Medical Association (2012) explicitly recommends within its social media guide not to accept friend requests on online communities from current or former patients.

\section{Features of domain-specific PSN for health workers}

Notwithstanding the previous mentioned concerns, a wide variety of PSN exist that are specifically designed for health workers and/or the healthcare domain. Table 1 shows a non-exhaustive list of extant health-related PSN. It is important to note that we only considered PSN that claim to be contextualized and that purpose to improve collaboration among them. We therefore did not include any social networking sites, such as PatientsLikeMe.com, which are rather meant for enhancing the patient-doctor-relationship or where health workers are not the primary end-user of the platform.

In order to analyze and represent commonalities and differences of the identified PSN, we distinguished general and domain-specific features. General features denote a cohesive set of functionality, which can be found in any kind of PSN, such as a personal profile, discussion boards, news boards, chats, or video and photo sharing. Domain-specific features represent a set of functionality, which are particularly designed for or are of unique interest to health workers like a medical case library or healthcare-specific job sites. Beyond some functional variances, the identified PSN also highly differ in terms of scope, access, and targeted user groups. While some platforms are open to health workers worldwide, we found that other platforms restrict their access to healthcare professionals, which are accredited or certified to treat patients in a particular national health system. Further limitations may also be imposed by the affiliation to a specific professional group. For instance, whereas some of the identified PSN are open to health workers of all 
specializations, others only granted access to a particular profession or career level, such as nurses, surgeons or medical students. Consequently, inter-professional and inter-career collaboration and knowledge exchange can strongly vary between the different PSN. However, broadly speaking it can be said that existing contextualized solutions often do not differ much from more general PSN, such as LinkedIn or Xing.

\section{Table 1. Overview of existing PSN for health workers}

\begin{tabular}{|c|c|c|c|}
\hline & \multicolumn{2}{|c|}{ Disciplinary focus } \\
\hline & & Professional group specific & Professional group unspecific \\
\hline \multirow{2}{*}{ 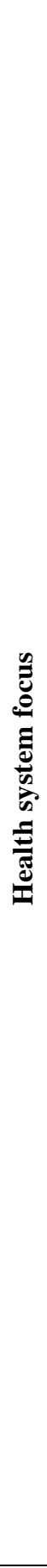 } & $\begin{array}{l}\frac{0}{3} \\
\frac{1}{3} \\
\frac{1}{3} \\
\frac{1}{3}\end{array}$ & $\begin{array}{l}\text { Allnurses.com: PSN designed for nurses and } \\
\text { nursing students } \\
\text { - General features: chat, discussion board } \\
\text { - Domain-specific features: job searches, } \\
\text { career advice } \\
\text { DoctorsHangout.com: PSN designed for } \\
\text { doctors and medical students } \\
\text { - General features: member page, posts, } \\
\text { discussion board, sharing of photos \& } \\
\text { videos, search features, event calendar } \\
\text { - Domain-specific features: medical library, } \\
\text { job searches } \\
\text { Orthomind.com: PSN designed for orthopedic } \\
\text { surgeons } \\
\text { - General features: member page, posts, } \\
\text { discussion board, news } \\
\text { Domain-specific features: product } \\
\text { reviews, medical library }\end{array}$ & $\begin{array}{l}\text { Docnet.com: PSN designed for healthcare } \\
\text { professionals world-wide } \\
\text { - General features: chat, discussion board } \\
\text { - Domain-specific features: market studies, } \\
\text { drug consultation } \\
\text { Medscape.com: PSN designed for healthcare } \\
\text { professionals world-wide } \\
\text { - General features: chat, discussion board, } \\
\text { - } \text { Dews } \\
\text { Neuros.org: PSN designed for healthcare } \\
\text { professionals, particularly medical students } \\
\text { - General features: chat, discussion board, } \\
\text { - } \text { sharing of photos }\end{array}$ \\
\hline & 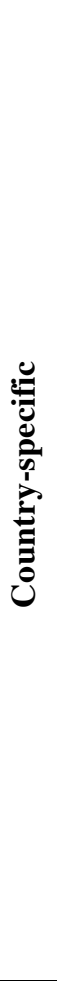 & $\begin{array}{l}\text { Nurse.com: PSN designed for U.S. nurses and } \\
\text { nursing students } \\
\text { - General features: news and blogs, event } \\
\text { calendar } \\
\text { - Domain-specific features: job searches, } \\
\text { store for nursing related shopping items, } \\
\text { links to webinar courses } \\
\text { Ozmosis.org: PSN designed for U.S. } \\
\text { physicians } \\
\text { - General features: chat, discussion board, } \\
\text { sharing of photos } \\
\text { - Domain-specific features: archive of } \\
\text { disease cases, social bookmarking } \\
\text { QuantiaMD.com: PSN designed for U.S. } \\
\text { physicians } \\
\text { - General features: chat, discussion board, } \\
\text { sharing of photos } \\
\text { Domain-specific features: contests, } \\
\text { archive of disease cases } \\
\text { Sermo.com: PSN designed for U.S. physicians } \\
\text { - General features: chat, discussion board, } \\
\text { sharing of photos }\end{array}$ & $\begin{array}{l}\text { MedicalMingle.com: PSN designed for U.S. } \\
\text { health professionals } \\
\text { - General features: member page, blogs, } \\
\text { posts, forum discussion, sharing of photos } \\
\text { \& videos, search features } \\
\text { - Domain-specific features: links to free } \\
\text { electronic journals, job searches, shopping } \\
\text { sites } \\
\text { medXcentral.com: PSN designed for U.S. } \\
\text { healthcare professionals } \\
\text { - General features: member page, blogs, } \\
\text { event calendar, posts, discussion board, } \\
\text { sharing of photos \& videos, search features } \\
\text { - Domain-specific features: None } \\
\text { MyMedPort.com: PSN designed for U.S. } \\
\text { healthcare professionals } \\
\text { - General features: member page, discussion } \\
\text { board, sharing of photos \& videos } \\
\text { - Domain-specific features: links to related } \\
\text { sites, marketplace for equipment, job } \\
\text { searches }\end{array}$ \\
\hline
\end{tabular}


- Domain-specific features: polls regarding the latest trends in healthcare

\section{An alternative perspective on contextualizing PSN}

The presented overview of existing platforms suggests that much of the PSN for health workers have instantiated a rather mechanistic perception of contextualization. Following the notion of domain engineering, which is primarily concerned with the engineering-forreuse or engineering-with-reuse of artifacts (Harsu, 2002; Reinhartz-Berger et al., 2013, Schmid, 2000; Tracz et al., 1993), contextualization is often reduced to a collection of artificial features (or solution components), which are configured, adapted, and made operative to solve specific problems of a particular business area or application (Kang et al., 1998).

However, while we share a pragmatist stance and likewise aim to generate constructive knowledge and useful artifacts for practice (Goldkuhl, 2012b), our understanding of domain engineering goes beyond a contemplation of configurable features and is possibly best conveyed by the work of evolutionary psychologists such as Chomsky (1980), Cosmides and Tooby (1989), or Hirschfeld and Gelman (1994).

According to this stream of research, human cognition and behavior is guided by a collection of innate domain-specific structures of knowledge (e.g. knowledge of language, knowledge of physical objects, and knowledge of number). Each domain builds its own way of perceiving and interpreting information and is unequally constrained by the format under which particular knowledge is represented (Carey and Spelke, 2008; Wellman and Berkowitz, 1988). For a new system to bring benefit to users, it is therefore crucial not only to know what are domain-related pressing problems and corresponding functional solutions, but importantly also how the members of a domain perceive and interpret their world and respond to it (Ellison and Boyd, 2013). To that effect, we comprehend domain engineering less as an approach to engineer or increase reuse of artifacts, but rather as a way to engineering-for-use such that an individual and his or her world-view, language, tradition, and context are well ingrained into the design of a new system (Brenner et al., 2014).

In pursue of a greater understanding why the identified domain-specific PSN fail to motivate health workers to seriously consider them for collaborative medical decisionmaking and knowledge exchange, we explored shared beliefs, attitudes, and expectations 
in prior research (blind for review). A summary of the most important reported issues and derived meta-requirements for a more domain-specific PSN are outlined in Table 2.

\section{Table 2. Key issues reported by health workers with existing PSN}

\begin{tabular}{|c|c|c|}
\hline Problem & Existing PSN & Meta-requirements for new PSN \\
\hline $\begin{array}{l}\text { Health workers avoid current PSN } \\
\text { because of fear of being spammed } \\
\text { by companies with marketing } \\
\text { messages or patients' friend } \\
\text { requests }\end{array}$ & $\begin{array}{l}\text { Restricted access based on } \\
\text { professional certification (e.g. } \\
\text { having a doctor's license) or } \\
\text { completely open to the public } \\
\text { - Harassment of health workers } \\
\text { by sales representatives and } \\
\text { companies still possible (e.g. } \\
\text { MD working at pharmaceutical } \\
\text { company) }\end{array}$ & $\begin{array}{l}\text { Boundary specification: } \\
\text { Provide for a common cultural } \\
\text { ground including shared goals, } \\
\text { negotiated meanings, and practices } \\
\text { that is safe from outside } \\
\text { interferences. }\end{array}$ \\
\hline $\begin{array}{l}\text { Health workers expect an } \\
\text { additional value or work-related } \\
\text { benefit from participating in a } \\
\text { PSN }\end{array}$ & $\begin{array}{l}\text { - Generic set of features (not } \\
\text { different from other social } \\
\text { networks) } \\
\text { - Building a sense of community } \\
\text { by granting access exclusively } \\
\text { to a specific professional group } \\
\text { Otherwise missing inter- } \\
\text { professional and inter- } \\
\text { organizational community } \\
\text { building features }\end{array}$ & $\begin{array}{l}\text { Community sentiment: } \\
\text { Create a strong feeling of } \\
\text { belonging together and sensation } \\
\text { of being part of something larger } \\
\text { such that participation and sharing } \\
\text { is fostered. }\end{array}$ \\
\hline $\begin{array}{l}\text { The targeted user group of } \\
\text { existing PSN is restricted to } \\
\text { professionals with superior } \\
\text { English skills and / or interest in } \\
\text { the U.S. health system }\end{array}$ & $\begin{array}{l}\text { Most PSN target an } \\
\text { international or U.S. audience } \\
\text { Language or culture related } \\
\text { differences are forgotten } \\
\text { Global content frequently does } \\
\text { not reflect actual interests and } \\
\text { needs of local health workers }\end{array}$ & $\begin{array}{l}\text { Culture \& language adaptation: } \\
\text { Incorporate local domain-specific } \\
\text { customs and language as well as } \\
\text { means for domain-specific } \\
\text { representation of knowledge. }\end{array}$ \\
\hline
\end{tabular}

\section{RESEARCH APPROACH}

Building upon the previous analysis, in this section we will describe how our metarequirements informed the development of various artifacts and organizational interventions that support our view on contextualization. In doing so, we followed an action design research (ADR) approach (Sein et al., 2011). The term 'action design research' was first mentioned by Iivari (2007) to refer to the combination of action research (AR) and design research (DR).

Like DR, ADR is motivated by the urge to design, use, and refine novel and useful artifacts (Hevner et al., 2004). The term 'artifact' is used to describe something that is artificial, or constructed by humans, in contrast to something that occurs naturally (Simon, 1996). 
Unlike DR, which strictly separates building from evaluating, ADR seeks to capture the emergent nature of the ensemble artifact and reflect both, anticipated and unanticipated designs. Similar to AR, organizational intervention and contextual factors play an important role in knowledge creation (Avgerou, 2001; Davison et al., 2004). While the researcher guides the initial design, the artifact emerges through the interaction between the development and use in context (Sein et al., 2011). This is typically an iterative process that aims to link theory with practice and thinking with doing (Susman, 1983). Two central assumptions of ADR are therefore (1) evaluation efforts cannot follow building in a sequence like in traditional stage-gate DR models, and (2) authenticity is more important than controlled settings. Since ADR combines practical problem solving with theoretical reflection, it has been advocated as a particularly viable approach for studying the design of socio-technical systems in healthcare (Scherer, 2014; Spagnoletti et al., 2015).

\section{Research context}

The results presented in this paper are the outcome of a five-year research project, which started 2009 and ended 2014 with the aim to improve collaboration between the health workers in the broader Lake of Constance area. In 2012, the population of this fast growing cross-boarder region-consisting of parts of Austria, Germany, Liechtenstein, and Switzerland-was nearly 3.9 million with an average population density of 290 persons per square kilometer.

The hospital landscape is characterized by the existence of 49 general hospitals both, public organizations owned and subsidized by local and regional authorities, and privateowned organizations which operate under for-profit models. Although there are a large number of cross-border movements (e.g. frontier commuter traffic and tourism), the delivery of health services is typically bound to the national territory. Knowledge exchange and communication between the regional hospitals has therefore been rather scarce, unsystematic, and idiosyncratic.

By means of an open call for participation, eight regional hospitals were recruited for this study, out of which three represent large general hospitals (or $20.0 \%$ of the total sample) with annually more than 9000 inpatient cases, and five rather medium or small hospitals 
(or $14.7 \%$ of the total sample) with typically less than 9000 impatient cases a year. ${ }^{1}$ Overall, the hospitals represent around 16.500 health workers, which voluntarily committed to take part in this ADR project (see Figure 1).

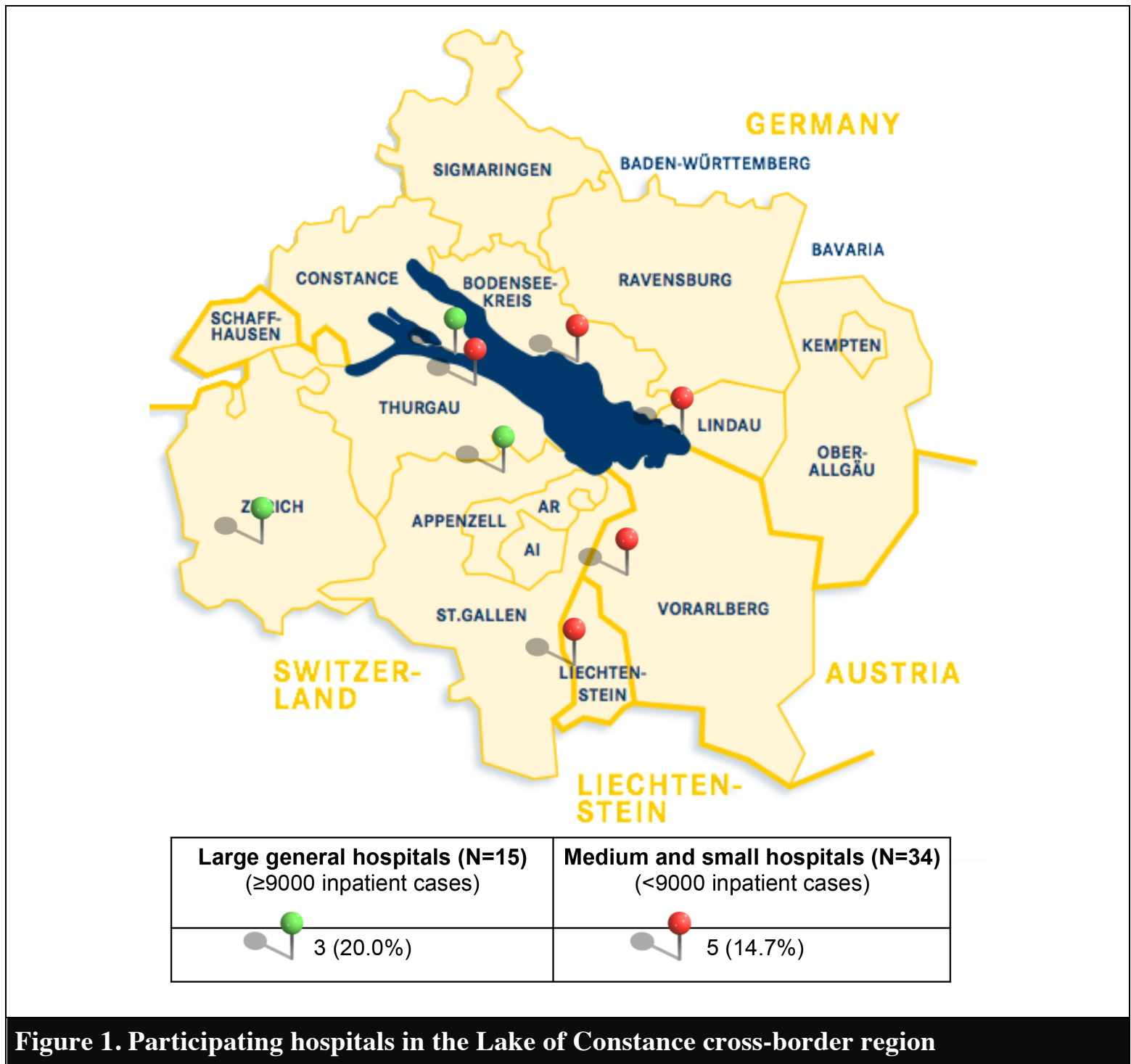

\section{Data collection and interventions}

Each hospital named a key contact person—typically the hospital director, chief physician, or chief nursing officer - that acted as gateway for providing access to the 'right' personnel in case of specific requests (e.g. to schedule interviews with specialized health workers, to get more in-depth information about the organization). Researchers and key contact persons formed the so-called ADR team (Sein et al., 2011).

\footnotetext{
1 Note: The classification of hospitals into large and medium-sized institutions has been based on the hospital typology
} developed by the Swiss Federal Statistical Office (2014). 
Facilitated by our access to a diverse group of health workers in different hospitals, in a first stage, we elicited general information about experiences and influencing factors that affect PSN-supported knowledge exchange and collaboration between health workers. In addition, we systematically collected ideas how our theory of contextualization could be practically realized and tested in our research context. In doing so, we used an interview guideline composed of a combination of focused and open-ended questions with regard to the identified meta-requirements on boundary specification, community sentiment, and culture and language adaptation (see previous section). The respondents' answers were transcribed verbatim a few days after the interviews and returned to them, so that a timely review of these transcriptions regarding their accuracy was guaranteed.

The data thereby obtained, on the one hand, corroborated the findings from prior research by acknowledging the fact that current PSN are not yet capitalizing their potential in improving knowledge exchange and medical education (Alkhateeb et al., 2011; Thackeray et al., 2012; Tunnecliff et al., 2015). On the other hand, the interviews provided us with valuable inputs for planning our subsequent actions and likewise confirmed our assumptions regarding the importance of having an adaptable, trustworthy, and safe environment for creating a community of practice.

Building upon the notion of IT-dominant building, intervention, and evaluation logic as described by Sein et al. (2011), our subsequent engagement was focused on employing early designs as light-weight interventions in a limited organizational context at first and then improve the design by continuously instantiating and repeatedly testing members' assumptions, expectations, and knowledge into the emerging artifact and widening the organizational setting to a broader group of end-users. An overall illustration of our iterative approach for developing our domain-specific PSN, which we named healthcare connect, is shown in Figure 2.

In line with Iivari (2015), we chose an approach that is focused on instantiating and deploying artificial designs into an organization since it is a priori better equipped to address immediate practical problems, but requires a stronger commitment of practitioners. It is thus noteworthy to mention that over a period of five years, more than 30 different health professionals regularly worked in close collaboration with us, yielding over 200 hours of interviews and focus group discussions taking place. $53.3 \%$ of the respondents 
were medical professionals (i.e. clinicians and nurses), 20.0\% technical support staff (i.e. IT-specialists, data managers), and $26.7 \%$ administrative staff or managers. ${ }^{2}$

In addition to the interviews and exploratory focus group sessions, we also gained insights from quarterly workshops with the ADR team. We used these meetings to discuss the actual progress of the artifact's building and evaluation activities as well as to plan prospective organizational interventions. It also helped us to establish an initial informal collaboration on institutional level and to collect and exchange ideas among all participating hospitals.

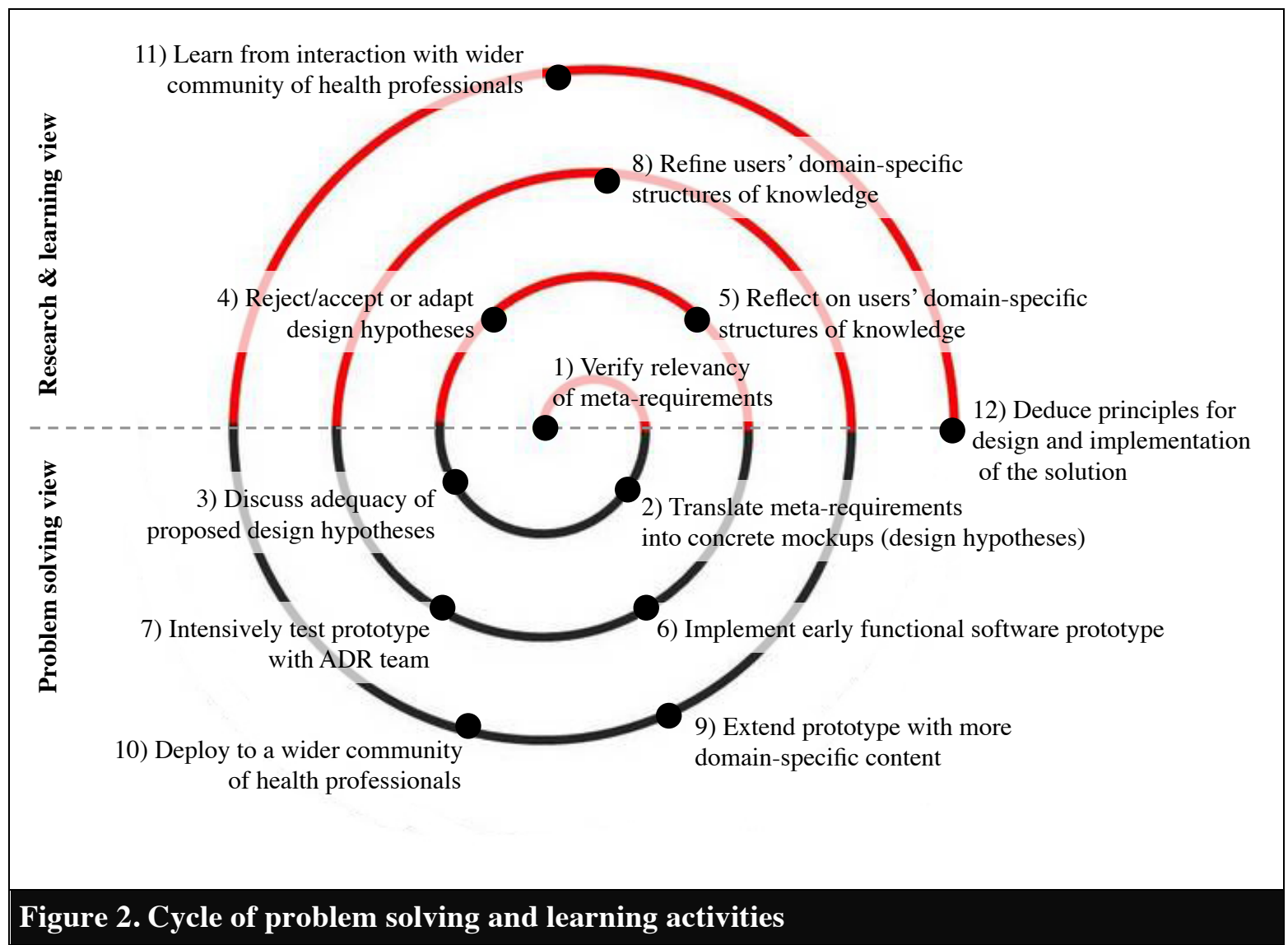

On the one hand, the intense and continual contact with health workers considerably eased problem solving, such as gradually defining, adapting, and instantiating our design hypotheses. Starting from rather vague meta-requirements, we were quickly able to sketch mockups and further concretize these initial design hypotheses to an early functional

2 Note: Our sample only minimally deviates from the distribution of job assignments in a common hospital in central Europe, which in Switzerland is 57.3\% medical professionals, 13.2\% technical support, and 29.5\% administration and management (Swiss Federal Statistical Office, 2014). 
prototype. On the other hand, it also contributed to a better perception of the users' domain-specific structures of knowledge, which allowed us to accept, reject, or adapt our initially formulated design hypotheses and to learn underlying mental models of health workers, which was key for reaching early acceptance of the solution.

\section{RESULTS}

The results of design-oriented research can take many forms, such as foundations or methodologies for rigorously building IT-based artifacts (Hevner et al., 2004; March and Smith, 1995), prescriptive guidelines for the sound design of organizational interventions and of management structures (Pries-Heje and Baskerville, 2008; Van Aken, 2005), or instantiations of the before mentioned outcomes (Vaishnavi and Kuechler, 2008).

In this section, we will present how we captured and managed concerns regarding the professional identity in the participating hospitals and how based on these findings we actually contextualized and instantiated domain-specific features of our healthcare connect PSN. ${ }^{3}$ We highlight three design features, which were particularly important for creating an inter-professional and inter-organizational community sentiment: (i) rules for privacyaware access regulation to PSN for health workers, (ii) an ontological semantic mapping mechanism for reducing language-related misinterpretations, and (iii) the introduction of user-generated content for creating added value to the community of practice. Since this "added value" may be perceived differently depending on the professional background and world-view of the end-user (Allen, 2005), we also explain possible tensions respectively outline alternative design decisions for each domain-specific feature of the PSN.

\section{Access rules for boundary regulation}

Considerable research exists that has examined why users contribute content (Wasko and Faraj, 2005) and how the existence of privacy mechanisms and access rules may increase trust and encourage the willingness to participate and share experiences and knowledge within online communities (Stutzman and Hartzog, 2012; Zheleva and Getoor, 2009). Most common social media applications therefore provide various access and control

\footnotetext{
3 Note: For a description of the technical implementation, please see Appendix A.
} 
mechanisms that allow or restrict certain individuals to access others' profiles and content without direct interaction with them (Ellison and Boyd, 2013; Gross and Acquisti, 2005).

Previous research (Grajales et al., 2014; Thompson et al., 2011) and our own evidence from the interviews with health workers indicated that there is a particularly great fear that the exchanged information on healthcare connect will be misused by other parties and/or exploited for other purposes than community building.

\section{Chosen resolution:}

Albeit it is possible to integrate multiple, less restrictive privacy features on different stages and levels for controlling access and usage (Child et al., 2012), a majority of the consulted health workers opted for a rather prohibiting solution that only grants access to employees of dedicated healthcare organizations, while leaving access to content, conversations, and groups in the PSN completely open once logged in. At first view, this problem seemed to be easy to solve. But going deeper, we had to take into account several unforeseen issues: Although a list of registered health professionals was available in all countries, it did not exclude professionals that worked in other organizational settings than healthcare. In addition, these registers only included doctors. All other health professionals working in healthcare organizations (e.g. nurses, technicians, administrators, managers) were not included in these lists. Accordingly, we started to experiment with different forms of access regulation mechanisms that were iteratively tested within the ADR team and refined over the course of the different design iterations. 


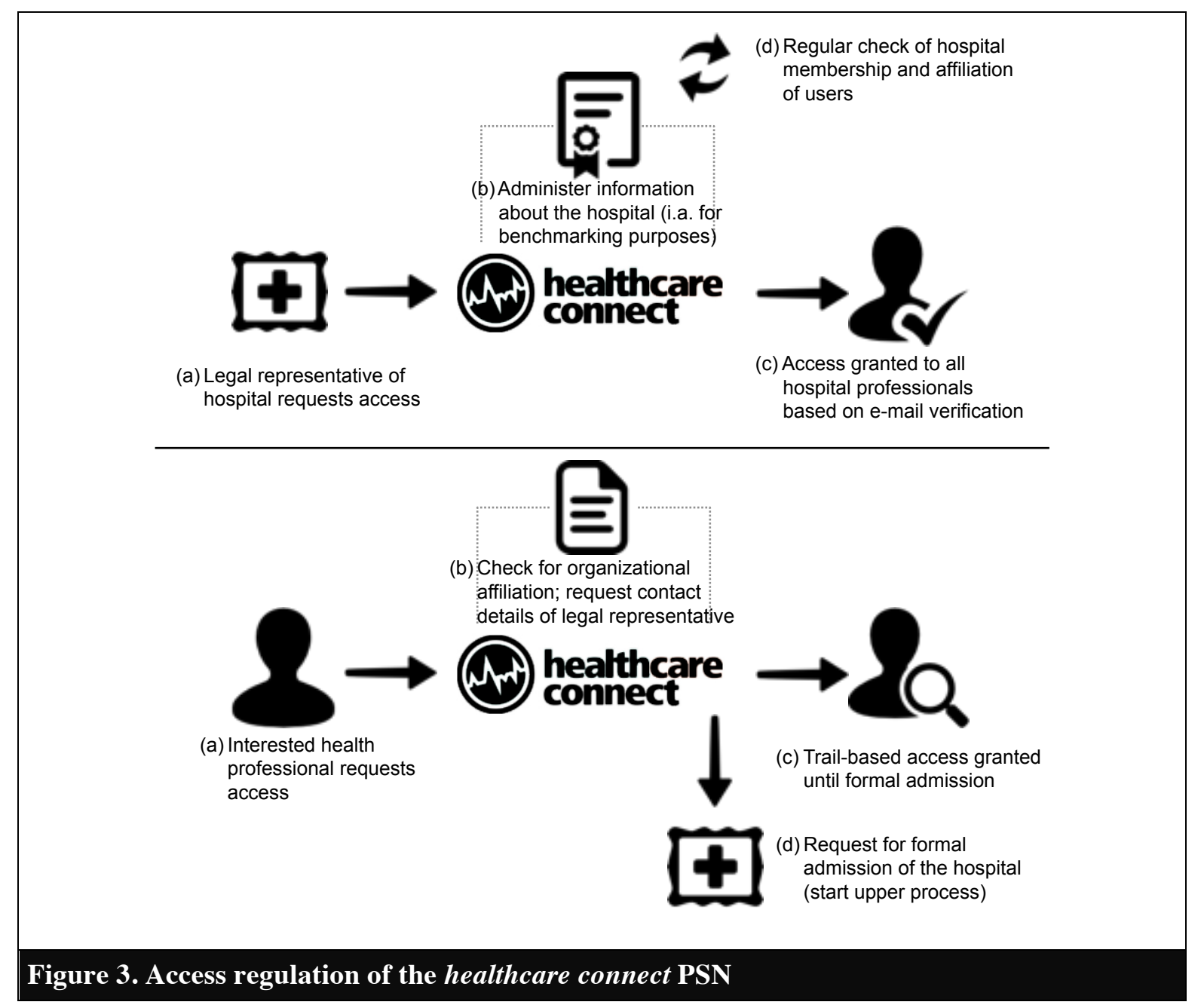

A central design decision was to tie access to healthcare connect to the organizational affiliation of a health professional. The ADR team followed the idea that only health professionals should be granted access to the platform if they are employed by a hospital, which is a member of the network. In this scenario (see upper part of Figure 3), interested professionals are granted access when a legal representative of the employing hospital has signed a participation contract and provided a minimal set of master data. Once the hospital is formally incorporated into the network, health workers who are interested in using the PSN can create an account by using their hospital e-mail address. A regular check of hospital membership and the affiliation of registered end-users are implemented to countervail possible fluctuations during the year (e.g. job change to a private enterprise). In the ongoing reflection and discussions within the ADR team, the opinion came up that it is more likely that an individual entity (and not a legal entity as stipulated first) would make the initial move. In order not to jeopardize the broadening of the network, it was 
decided to also grant access to health workers on a trial basis (see lower part of Figure 3). While it is still possible that the interested health worker is excluded from the PSN by hindsight, it nevertheless offers an interested health professional the opportunity to explore the platform and to see if a participation could be of value or not. This measure generally diminished the level of skepticism and reactions to the final version of healthcare connect were consistently positive.

\section{Tensions with the instantiated design:}

While for most of the consulted health workers the presented solution seemed to avert their biggest threat of having outsider interference in the PSN, a minority of administrators and physicians also desired more granular access rules including, for example, restricted areas for dedicated occupational groups (e.g. doctors' lounge), institutions (e.g. private space of hospital $x$ ), or even departments (e.g. billboard for department $y$ ). Although technically this would have been solvable and possibly increased acceptance of the aforesaid respondents, we decided against the introduction of further restrictions as it might have jeopardized our goal of increasing inter-organizational cooperation between the institutions in the crossborder region and the inter-professional collaboration among the diverse occupational groups in these hospitals. In this sense, a major tension not only presents the extent to which the PSN is open or restricted for outsiders of the profession, but also the extent to which it allows encapsulated forms of use-as would have been the creation of isolated spaces for distinct occupational groups, departments, or institutions in our case.

\section{Ontological semantic mapping for reducing language problems}

Reasonably strong evidence exists that sharing a common language and evolving a common set of values and objectives is a major precondition for an online community to prosper (Choi et al., 2013; Sherif et al., 2006). Particularly in expert domains, such as medicine, where precise communication is critical to effective behavior and successful service delivery, divergent local cultures and understandings may cause major problems of coordination (Walsh and Maloney, 2007) and eventually prevent the development of a community sentiment (Yamashita et al., 2009).

Many of the consulted health workers remarked that they felt uneasy to use a PSN in a foreign language for collaborating with each other. The discussions also revealed that even when using the same native language (in this case German) there was a considerable risk 
of misunderstanding, since the professional language varied to some extent (e.g. different names for conditions, materials, or institutions) in the different countries of the Lake of Constance region.

\section{Chosen resolution:}

To support the emergence of domain-specific structures of knowledge and to reduce confusion and misinterpretation in daily work, the ADR team started to iteratively develop an ontological foundation of key terms (cf. Figure 4).

Depending on the location of the end-user, this ontology allowed us to display frequently used terms according to local customs. Moreover, it also facilitated the development of other services provided on the platform (e.g. organizational or group benchmarks), without which it would have been impossible to do so. Admittedly, we could not cover the entire complexity and variability of the professional language of the distinct countries. But reactions from both the ADR team and end-users were positive and highlighted the importance of having some kind of semantic mapping, especially if it is the aim to improve inter-organizational collaboration in a multi-cultural context.

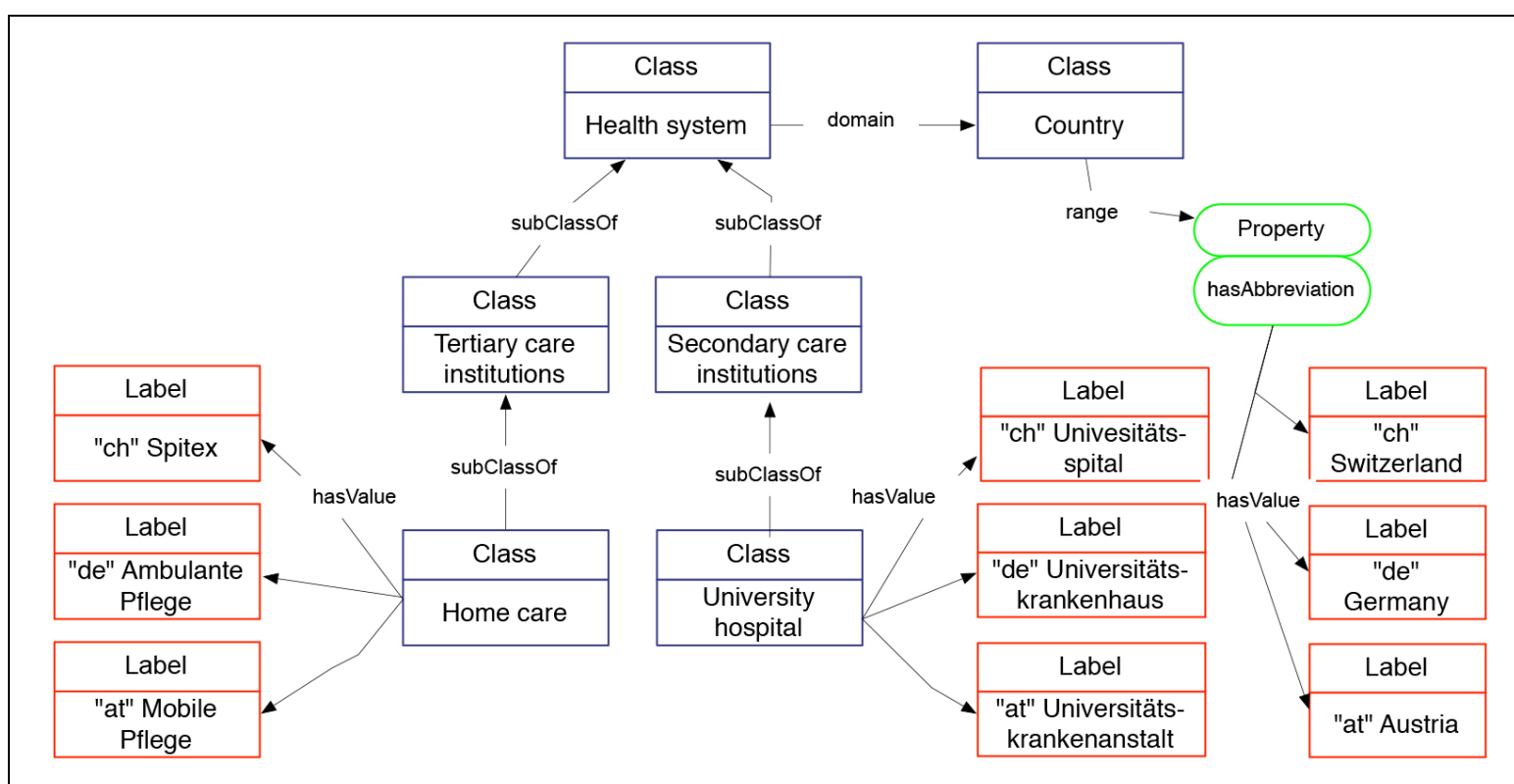

Figure 4. Excerpt of semantic mapping of country-specific professional terms

Tensions with the instantiated design:

While the semantic mapping and language adaptation was perceived to be useful for enhancing collaboration among health workers from different country-contexts, there have 
also been respondents who preferred to conserve the local idioms so as to be more efficient in their communication. Since the semantic mapping is not applied to conversations within the PSN, we could not really empathize with this concern. However, it points to a delicate issue: Is language adaptation really necessary in PSN or should we rather conserve the local jargon of distinct occupational groups? There is no simple answer to this question as it depends on the superior goal one wants to achieve with a contextually anchored PSN. Since we particularly wanted to improve inter-organizational and inter-professional exchange, we opted for a solution that perhaps decreases the efficiency within established groups, but improves precision of communication among various members of the PSN. However, the decision to adapt or conserve local domain-specific customs, language, and knowledge is problematic within a contextualized PSN.

\section{User-generated and domain-specific content for increasing added value}

The biggest challenge presented the provision of regular, interesting, and relevant content to the platform. Several studies have indicated that this is a major precondition for creating a community sentiment and contagion effect in social media applications (Aral and Walker, 2011; Bampo et al., 2008).

The lack of health-related content in an earlier version of healthcare connect was found to be a major issue by health workers as they seemed to have problems picturing how the final PSN could look like, what kind of information and features would be available, which professional and inter-professional groups would be active on the platform, and finally, what ultimate added-value of using this platform would result for them.

Accordingly, we received extremely mixed reactions to our proposed design at the beginning of our ADR project. While one group of health workers only acknowledged the need for domain-specific content, another group of users proposed a detailed catalog of functional, expressive, and aesthetic measures that caused a major redesign of healthcare connect. ${ }^{4}$ These reactions indicate that providing an "empty shell", even in a test phase, is problematic as it creates confusion and disbelief among potential users regarding the usefulness and added value of having an online community of practice.

\footnotetext{
4 Note: For an example of a major design shift from the first prototype to the final design of the PSN, please see Appendix B.
} 


\section{Chosen resolution:}

A number of measures were initiated to breathing new life into our PSN including, for example, teaming up with media companies specialized in the healthcare domain to fill the news section of the platform, bringing various health workers and associations to use healthcare connect for managing their group activities (cf. Figure 5d), motivating them to plan group events (cf. Figure 5b), writing (or moving their existing) blogs to the opinions and dialog section, or posting tech reviews and conducting short polls. We even included other artifacts, such as benchmarks (cf. Figure 5c), which we developed in previous research. Throughout this enrichment process, particular emphasis was given to identifying topics that are equally interesting for a large group of health workers. This been specially appreciated by those health workers, who had the sensation to be excluded from the rather specific discussions in occupational sub-disciplines.

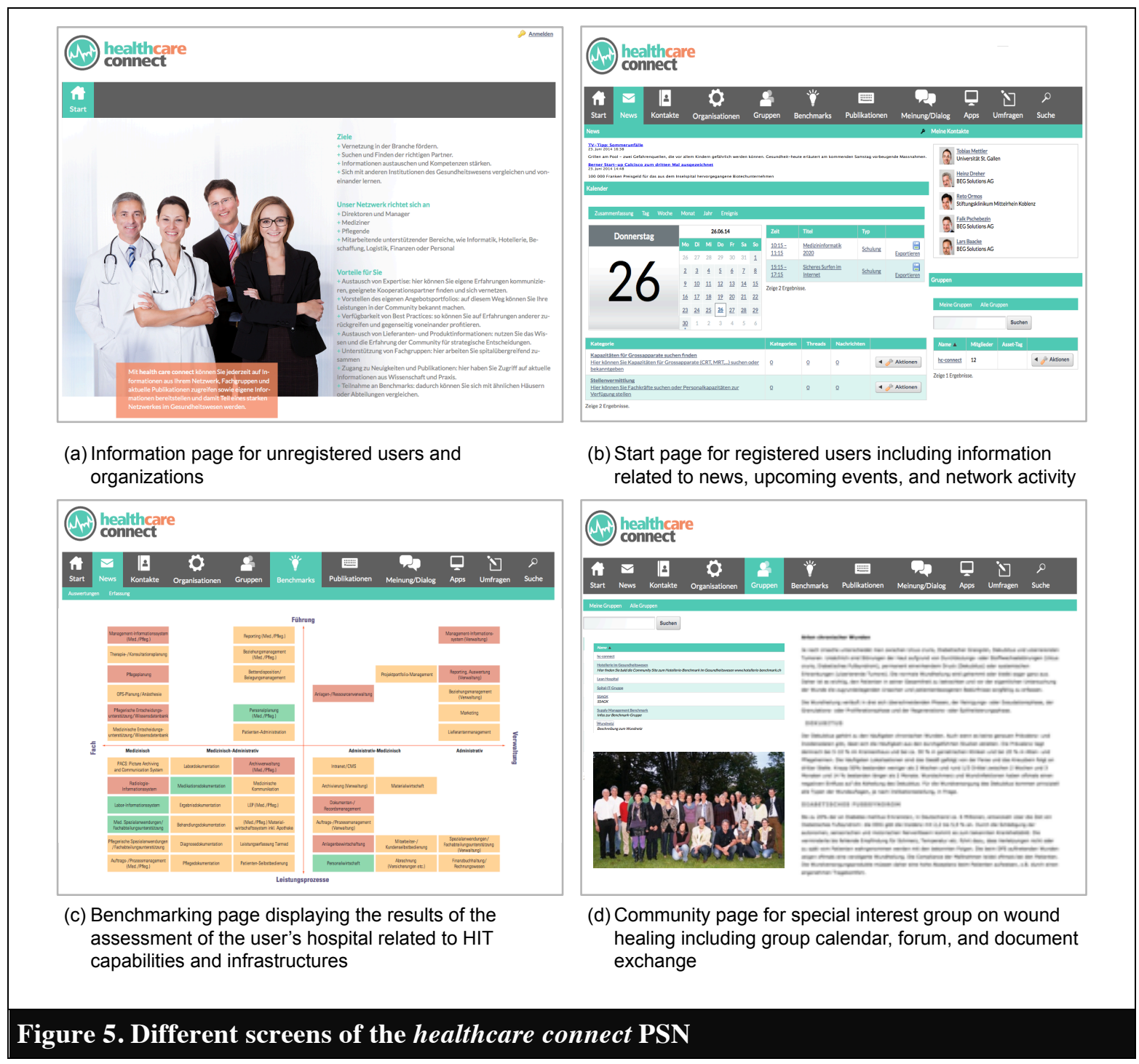




\section{Tensions with the instantiated design:}

Our approach to create community sentiment by means of a common content base in the PSN was not likewise appreciated by all respondents. Accordingly, we identified a tension between accentuating mutual interests and providing specialized content areas. Investigating this tension further, we found that the differences in value judgments could not only be explained by the simple fact that distinct occupational groups perceive certain of our identified topics more or less interesting. Rather, additional factors such as career level (e.g. missing contents suitable for residents) or the extent to which an end-user is focused on national health policy (respectively also interested in questions concerning supra-regional developments) also explained the different reactions to our content. The fact that the profession is not the determining factor for adapting IT to the health context is consistent with prior research (Mettler, 2013).

\section{DISCUSSION}

The presented findings show an engaged and transformative style of research that is framed within a pragmatic philosophy (Mathiassen and Nielsen, 2008). As such, contributions to the knowledge base are not necessarily restricted to explanations and to understanding, but can also have normative or practical character (Goldkuhl, 2012a; Gregor and Hevner, 2013). The contribution of this paper is therefore twofold. In the next subsection, we will first describe the key learning from a practical perspective, which is derived from our observations how health workers interacted with healthcare connect after implementing the final design of the PSN. In the subsection thereafter, we will employ metaphors as vehicles for complementing the practical learning with narrative thinking (Venable, 2006) and for stimulating the reflection from a theoretical point of view (Hirschheim and Newman, 1991; Iivari, 2007).

\section{Practical implications}

As a large number of previous research studies have shown (Amoako-Gyampah and White, 1993; Bano and Zowghi, 2015; Ives and Olson, 1984) that early user involvement has proven to be extremely helpful in identifying needs, priority setting, and designing and evaluating the design of our platform. Nevertheless, it is frequently the case that users interact with the artifact in for the designer unforeseen ways, such as going to the extreme of temporarily or even permanently developing workarounds for overcoming capability 
limitations of the developed system or bypassing existing organizational practices (Alter, 2014; Ferneley and Sobreperez, 2006). During a test phase of six months, where we deployed and introduced healthcare connect to the participating hospitals, we observed both anticipated and unanticipated uses of our PSN, which are summarized in Table 3 and which we discuss in the following.

\section{Table 3. Intended, unintended, and unfulfilled use of healthcare connect}

\begin{tabular}{|l|l|}
\hline Intended use & - Establishing inter-organizational relationships \\
& $\begin{array}{l}\text { - Accessing news and articles from professional content providers } \\
\text { - Disseminating promotional material for and organization of professional events } \\
\text { - Exchanging general best practices and benchmarking selected operational areas }\end{array}$ \\
\hline $\begin{array}{l}\text { Intended but } \\
\text { unfulfilled use }\end{array}$ & $\begin{array}{l}\text { - Establishing inter-professional relationships } \\
\text { - Developing knowledge base for ensuring evidence-based continuing education }\end{array}$ \\
\hline $\begin{array}{l}\text { Unintended use } \\
\text { or workaround }\end{array}$ & $\begin{array}{l}\text { - Posting of open positions and recruiting of health professionals } \\
\text { - Using functionalities for purposes other than work as proxy for other blocked social } \\
\text { media applications }\end{array}$ \\
\hline
\end{tabular}

Intended use of designed PSN. In view of the supra-regional character of this ADR project, it was a major objective to build a platform that helps establishing inter-organizational relationships among health workers in a natural an easy manner. We were therefore glad to see that many new connections of user profiles between the different participating hospitals were established during the test phase. We believe that the regular interaction with health workers was not only instrumental for eliciting and validating functional and aesthetic end-user needs, but also an important catalyst for identifying opinion leaders and promoters who supported us to advertise our solution among their colleagues. We were also happy to observe that our efforts in involving professional content providers were largely utilized by the members of the PSN. Accessing and discussing news and articles emerged to be one of the major use cases of healthcare connect. The PSN was also regularly exploited for disseminating promotional material, to inform colleagues about upcoming events, or for planning and organizing professional conferences together. To a lesser extent, healthcare connect was also used to exchange general best practices. However, it seemed that knowledge creation and retention was not a main driver for using the PSN. The reasons for that could be the existence of other medical knowledge repositories in the hospitals, distinct national or regional regulations that hinder to develop 
a common ground, unknown credentials of the contributors, or apprehension of publishing poor content.

Intended but unfulfilled use of designed PSN. While we were glad to see growing interorganizational connections, at a closer look, we found that most of the relationships were evolving within the same occupational group (e.g. administrators, doctors, and nurses of different hospitals). Accordingly, our goal to particularly improve inter-professional exchange was not fully achieved with the current design. As we explained earlier, creating community sentiment by means of accentuating common interests has been controversial. Sadly, we found that current professional structures and world-views still impede the establishment of new forms of collaboration and create inflexibility in modifying such arrangements to adapt to changing conditions (Kvarnström, 2008; San Martín-Rodríguez et al., 2005).

Together with an improved exchange of best practices, we also wished for stronger collaboration for solving and sharing experience about challenging cases and for developing a mutual knowledge base for ensuring evidence-based continuing education (King et al., 2009; Sanders and Schroter, 2007). Already at the beginning of the project we knew that information sharing in general, and regarding dedicated patient cases in particular, is significantly affected by privacy concerns and hospital information policies (Fichman et al., 2011; Leonardi, 2014) - even when all data sets are anonymized! Accordingly, developing reasonable notions of privacy concerning the use of PSN in hospitals is an important task for the future.

Lastly, in order to fill the gap of required but unavailable skilled personnel as well as to support foreign health workers in quickly getting immersed into the unknown social microstructure of the hospital and health system (Kamel Boulos and Wheeler, 2007), another intended use of our platform was to support recruiting and talent management. While this has been a top priority of all involved parties, it also led to many discussions. Due to inequalities of national minimum wages and working conditions at the hospitals, some hospital directors feared a severe brain drain to competitors across the border. Others expressed concerns because they were worried that the PSN will deteriorate to a platform for headhunters and professional recruiters. In consequence, although prior studies have predicted a rise of employing social media for recruiting talents (Amankwah-Amoah and Sarpong, 2014), our efforts could not corroborate this potential benefit. 
Unintended use and workarounds. During the test phase, we also observed how health workers interacted with our PSN in unintended ways. While in some cases workarounds and unintended uses lead to unforeseen, positive impacts in organizations, this was not the case in our study. Although we repeatedly emphasized the inter-professional and interorganizational character of healthcare connect, in some cases end-users employed our PSN not necessarily to widen their disciplinary and institutional scope, but rather as informal channel or "shadow IT" system (Silic and Back, 2014) for internally organizing themselves or for discussing highly specialized topics, which were not suitable for a larger audience. In this sense, our PSN served as substitute for unavailable or inadequate coordination mechanisms within the departments or hospitals. This could be problematic as considerable amount of time and effort could be misspent for keeping the formal infrastructure, such as care planning and clinical management systems, up-to-date and compliant with organizational norms. In addition, a couple of health workers used healthcare connect for purposes other than work, such as for private conversations with colleagues since other social media applications were blocked. Accordingly, not only inefficiencies in allocation and utilization of IT resources could be created, but also the risk of workarounds that bypass organizational control and information policies.

\section{Theoretical implications}

As we noted earlier, traditional approaches to domain engineering are frequently concerned with engineering-for-reuse or engineering-with-reuse of artifacts instead of focusing on engineer-to-use, respectively how the members of a particular domain perceive, interpret, and respond to a proposed solution. Accordingly, existing approaches to domain engineering are often mechanistic and lack a pluralistic view on identity and community building. Especially for the healthcare domain, it was found that culture and language adaptation, community sentiment, and boundary specification are important meta-requirements for a PSN. In operationalizing these meta-requirements by different solution components (i.e. rules for privacy-aware access, ontological semantic mapping, user-generated enrichment) we not only gained practical experience so as to understand intended, unintended, and unfulfilled uses of our proposed design, but also found narrative interpretations and metaphors how to understand domain engineering in an alternative way, which we describe next. 
Domain engineering as problem solving. Design-oriented research is inherently problemdriven (Holmström et al., 2009). In this sense, from a mechanistic point of view, developing a domain-specific PSN may be understood as the identification, analysis, and adaptation of general and/or development of new features in order to comply with the needs of the members of a domain (Santos de Oliveira and Gerosa, 2011). However, besides solving engineering-related problems, our ADR approach also allowed us to gain richer insights into multi-faceted and more profound organizational and cultural issues. For instance, in several of the participating hospitals we encountered the situation that an overall information policy was missing or not specified in such detail so that we knew what kind of information and how much of it can be disclosed in the PSN. Often health workers also approached us with "soft” or "wicked” problems (Rittel and Webber, 1973), such as providing a solution for changing deadlocked perceptions on occupational hierarchy, niche behavior or local thinking. Although this initially seemed to be completely unrelated to the goals of the project, we realized with the advancement of the ADR process that many of the raised concerns affected acceptance of the PSN. While with our engaged research process we could not resolve all the conflicts and omissions in the hospitals, we highly advise taking domain engineering as an opportunity for system thinking and higherorder problem solving as opposed to a pure feature-driven or re-use focused approach to engineer artificial solutions and adapt them to the needs of a domain.

Domain engineering as collective action. Since developing a PSN is about social interaction and the creation of a sense of community (Carter, 2005; Ellison and Boyd, 2013), we may also understand domain engineering - in this particular case-as collective action. As we discussed earlier, simply providing an "empty" artifact does not convey this sentiment. Only if developers are able to motivate potential end-users to share their ideas and world-views, to define their language patterns, and to actively and regularly contribute content and engage with others it is possible to create an added value for a larger, yet reluctant group of users and to breath life into the PSN. In this sense, domain engineering may only be successful if it is realized as cooperative and mutual problem solving and learning process between open-minded developers and engaged members of the domain.

Domain engineering as form of control. In order that a strong community sentiment can emerge, a balance between inclusiveness and exclusiveness is required (Naylor et al., 1996). Particularly in expert domains, such as medicine, this exclusiveness is attained by using a sophisticated language and mental representations, organizing knowledge in a 
domain-specific way, and establishing clear principles of professional conduct (Cnossen, 2014). Accordingly, by reproducing these structures of knowledge and communication, developers may (intentionally or unintentionally) govern the level of non-experts to enter the domain. As we have shown with our access regulation rules, there are also immediate possibilities to restrict participation to undesirable individuals and/or organizations. Hence, we frequently had the sensation that domain engineering is also a form of controlling social interaction and access to the community of practice.

Domain engineering as cultivation of the installed base. Finally, an engaged approach to domain engineering may also serve as a means through which it is possible to deliberate about intentional and emergent, or unexpected designs. Many studies have shown that, especially in healthcare, there is the phenomenon of unintended or unplanned "solution engineering" by health workers (Aanestad and Jensen, 2011; Grisot and Vassilakopoulou 2013). We share this perception, as we were often confronted with situations in which potential end-users adopted unintentional usage patterns or expressed ideas outside our initial project scope. While the analysis, implementation, and further discussion of these unplanned use cases was extremely laborious, we highly recommend allocating enough time to the project such that end-users can develop unintentional usage behavior and such that it is possible to transfer these experiences into the intended design of the PSN.

\section{CONCLUSION}

This research was motived by the question of how to capture and manage the professional identity of an industry such that a PSN can be purposefully anchored in the working context. By adopting ADR as an engaged and transformative style of conducting research, we have demonstrated that contextualization of healthcare connect - a PSN specifically design for improving inter-professional and inter-organizational exchange in hospitals - is a complex undertaking that requires both problem solving and reflection germane to technological, but also to organizational and cultural aspects.

Since our research is built upon the premises and postulates of design-oriented research, we need to demonstrate that we conducted a rigorous evaluation (Gregor and Hevner, 2013) and produced new generalized knowledge about, and with design (Baskerville, 2008). However, ADR is different from customary design-oriented research because it understands artifact construction not as a separable sequence of build-and-evaluate 
activities (Peffers et al., 2008; Walls et al., 1992), but rather as an emergent and contextsensitive iterative process without clear lines between designing and testing as well as the organizational environment and the IT artifact itself (Sein et al., 2011).

Having said this, we validate our research by contrasting it with Hevner's et al. (2004) seven guidelines, which are frequently used as directives for the assessment of the validity, utility, and quality of design-oriented research.

(1) Research outcome is a viable artifact. We present an IT-reliant PSN, which we named healthcare connect. Our artifact includes three instantiated solution components (i.e. rules for privacy-aware access, ontological semantic mapping, user-generated enrichment) that are instrumental for tailoring a PSN towards the domain-specific needs of health workers.

(2) Research produces solutions to relevant business problems. Improved collaboration and knowledge exchange is vital for complex service industries, such as healthcare. PSN help health workers to quickly get immersed into the social microstructure of an organization and community as well as support the learning of region-, organization-, or group-specific (tacit) practices and knowledge that is required for delivery a high-quality service.

(3) Research demonstrates utility, quality, and efficacy. Our research is valuable in that we developed a domain-specific PSN that was tested over a longer period of time in eight regional and general hospitals in the Lake of Constance region. Despite the fact that our solution is not yet in production use (i.e. not rolled-out to all end-users), involved health workers attested a positive influence on improved collaboration within and across the participating organizations.

(4) Research contributes to design foundations and/or methodologies. Besides building a practical artifact, we also contributed to the knowledge base in two ways. First, we derived design principles that may serve as a foundation for guiding the development and implementation of domain-specific PSN. Second, we identified metaphors as vehicles for increasing our understanding on domain engineering.

(5) Research is conducted in a rigorous manner. Our research is based on prior evidence from the wide area of social media and rooted in the philosophy of design-oriented research. Having ADR as our overarching research method, we followed both the standards of design-oriented and qualitative research, including - amongst others - efforts to control for retrospective or key informant bias or systematic artifact construction. 
(6) Research is performed as a problem-situated means-ends search for an effective artifact. Starting from meta-requirements (problem space) and a review of the functionality of currently available PSN (solution space), our research has followed an iterative cycle of practical problem solving and theoretical reflection.

(7) Research is communicated to both technical and managerial audiences. We postulate that for the design of domain-specific PSN a co-evolutionary approach is required, which combines the technical with the social perspective of domain engineering. We recommend using an engaged style of research that not only consists of technical design activities but also includes collective action by means of organizational interventions for introducing PSN into the intended environment of usage.

Our research certainly is limited in a number of respects. First, the focus of this study has primarily been on domain engineering and the contextualization of PSN. At this point, however, little is known about how end-users effectively appropriate value from using PSN and how this is compatible with organizational norms, intellectual property rights, and other forms of organizational control. While we provide first insights of how health workers interact with a contextualized PSN, there is an inherent need for further research that describes practical real-life examples and integrates long-term user experiences beyond an initial test run. We therefore seek to continue to explore intended and unintended uses once healthcare connect is operational in the near future. Second, the healthcare domain and the organizational contexts in which this study was conducted entail various specific characteristics, which certainly influenced the identified design propositions and metaphors. Even though we made every effort to be exhaustive in our contextual description and highlighting the interdependence between the IT artifact and context, transferring our findings to other industries should be handled carefully and be extended by experiences from other domains. Last but not least, our study did not elaborate in detail on the important issue of how to adapt a PSN so that a contagion effect can be achieved. This is a fundamental problem in every network structure-digital or physical. Although this has not been the main focus of this study, it could represent a new and exciting avenue for future research particularly due to the fact that PSN are different from privately used social media applications since companies could either restrict or promote their adoption in the organizational environment. 


\section{REFERENCES}

Aanestad, M. and Jensen, T. B. (2011) Building Nation-Wide Information Infrastructures in Healthcare through Modular Implementation Strategies, The Journal of Strategic Information Systems 20 (2): 161-176.

Alkhateeb, F. M., Clauson, K. A. and Latif, D. A. (2011) Pharmacist Use of Social Media, International Journal of Pharmacy Practice 19 (2): 140-142.

Allen, J. P. (2005) Value Conflicts in Enterprise Systems, Information Technology \& People 18(1): 33-49.

Alter, S. (2014) Theory of Workarounds, Communications of the Association for Information Systems, 34(1): 1041-1066.

Amankwah-Amoah, J. and Sarpong, D. (2014) The Battle for Brainpower: The Role of Market Intermediaries in Lateral Hiring, Strategic Change 23 (3-4): 237-251.

American Medical Association New Ama Policy Helps Guide Physicians' Use of Social Media [WWW document] http://www.ama-assn.org/ama/pub/news/news/social-mediapolicy.page (accessed September 3, 2015).

AMN Healthcare (2013) 2013 Survey of Social Media and Mobile Usage by Healthcare Professionals, San Diego, USA: AMN Healthcare.

Amoako-Gyampah, K. and White, K. B. (1993) User Involvement and User Satisfaction: An Exploratory Contingency Model, Information \& Management 25 (1): 1-10.

Anderson, R. M. and Funnell, M. M. (2005) Patient Empowerment: Reflections on the Challenge of Fostering the Adoption of a New Paradigm, Patient Education and Counseling 57 (2): 153-157.

Aral, S. and Walker, D. (2011) Creating Social Contagion through Viral Product Design: A Randomized Trial of Peer Influence in Networks, Management Science 57 (9): 16231639.

Argyris, Y. A. and Monu, K. (2015) Corporate Use of Social Media: Technology Affordance and External Stakeholder Relations, Journal of Organizational Computing and Electronic Commerce 25 (2): 140-168.

Avgerou, C. (2001) The Significance of Context in Information Systems and Organizational Change, Information Systems Journal, 11 (1): 43-63.

Bampo, M., Ewing, M. T., Mather, D. R., Stewart, D. and Wallace, M. (2008) The Effects of the Social Structure of Digital Networks on Viral Marketing Performance, Information Systems Research 19 (3): 273-290. 
Bano, M. and Zowghi, D. (2015) A Systematic Review on the Relationship between User Involvement and System Success, Information and Software Technology 58 (2): 148169.

Baskerville, R. (2008) What Design Science Is Not, European Journal of Information Systems 17 (5): 441-443.

Brenner, W., Karagiannis, D., Kolbe, L., Krüger, J., Leifer, L., Lamberti, H.-J., Leimeister, J., Österle, H., Petrie, C., Plattner, H., Schwabe, G., Uebernickel, F., Winter, R. and Zarnekow, R. (2014) User, Use \& Utility Research, Business \& Information Systems Engineering 6 (1): 55-61.

Bricon-Souf, N. and Newman, C. R. (2007) Context Awareness in Health Care: A Review, International Journal of Medical Informatics 76(1): 2-12.

British Medical Association Social Media Use: Practical and Ethical Guidance for Doctors and Medical Students [WWW document] http://bma.org.uk/-/media/files/pdfs/practical advice at work/ethics/socialmediaguidance.pdf (accessed September 3, 2015).

Bruno, G., Dengier, F., Jennings, B., Khalaf, R., Nurcan, S., Prilla, M., Sarini, M., Schmidt, R. and Silva, R. (2011) Key Challenges for Enabling Agile BPM with Social Software, Journal of Software Maintenance and Evolution: Research and Practice 23 (4): 297-326.

Cain, J. (2008) Online Social Networking Issues within Academia and Pharmacy Education, American Journal of Pharmaceutical Education 72 (1): 1-7.

Cameron, A. F. and Webster, J. (2005) Unintended Consequences of Emerging Communication Technologies: Instant Messaging in the Workplace, Computers in Human Behavior 21 (1): 85-103.

Camlek, V. (2015) Professional Medical Social Networks: An Evolving Source of Professional Knowledge and Content, Information Services \& Use 35(1-2): 77-87.

Carey, S. and Spelke, E. (2008) Domain-Specific Knowledge and Conceptual Change, in Adler, J. E. and Rips, L. J. (eds.) Reasoning - Studies of Human Inference and Its Foundations, Cambridge: Cambridge University Press, pp. 807-826.

Carter, D. (2005) Living in Virtual Communities: An Ethnography of Human Relationships in Cyberspace, Information, Community and Society 8 (2): 148-167.

Chiasson, M. W. and Davidson, E. (2004) Pushing the Contextual Envelope: Developing and Diffusing IS Theory for Health Information Systems Research, Information and Organization 14 (3): 155-188. 
Chiasson, M. W., Davidson, E. (2005) Taking Industry Seriously in Information Systems Research, MIS Quarterly 29(4): 591-605.

Child, J. T., Haridakis, P. M. and Petronio, S. (2012) Blogging Privacy Rule Orientations, Privacy Management, and Content Deletion Practices: The Variability of Online Privacy Management Activity at Different Stages of Social Media Use, Computers in Human Behavior 28 (5): 1859-1872.

Choi, J., Lev, B. and Kim, H.-L. (2013) Exploring Determinants of Knowledge Sharing in a Social Network of Practice, in Proceedings of the 7th International Conference on Management Science and Engineering Management (Philadelphia, PA, 2013), pp. 3951.

Chomsky, N. (1980) Rules and Representations, New York: Columbia University Press.

Cleven, A. K., Winter, R., Wortmann, F. and Mettler, T. (2014) Process Management in Hospitals: An Empirically Grounded Maturity Model, Business Research 7 (2): 191216.

Cnossen, F. (2014) Cognitive Skill in Medicine: An Introduction, in Lanzer, P. (ed.) Panvascular Medicine, Berlin, Heidelberg: Springer pp. 1-41.

Cosmides, L. and Tooby, J. (1989) Evolutionary Psychology and the Generation of Culture, Part II. A Computational Theory of Social Exchange, Ethology and Sociobiology 10 (1-3): 51-97.

Cross, R., Borgatti, S. P. and Parker, G. (2003) Making Invisible Work Visible: Using Social Network Analysis to Support Strategic Collaboration, California Management Review 44 (2): 25-46.

Davison, R. M., Martinsons, M. G. and Kock, N. (2004) Principles of Canonical Action Research, Information Systems Journal 14 (1): 65-86.

Ellison, N. B. and Boyd, D. (2013) Sociality through Social Network Sites, in Dutton, W. H. (ed.) The Oxford Handbook of Internet Studies, Oxford, UK: Oxford University Press, pp. 151-172.

Epstein, S. R. (1998) Craft Guilds, Apprenticeship, and Technological Change in PreIndustrial Europe, The Journal of Economic History 58 (3): 684-713.

Ferneley, E. and P. Sobreperez (2006) Resist, Comply or Workaround: An Examination of Different Facets of User Engagement with Information Systems, European Journal of Information Systems, 15 (4): 345-356. 
Fichman, R. G., Kohli, R. and Krishnan, R. (2011) The Role of Information Systems in Healthcare: Current Research and Future Trends, Information Systems Research 22 (3): 419-428.

Goldkuhl, G. (2012a) From Action Research to Practice Research, Australasian Journal of Information Systems 17 (2): 57-78.

Goldkuhl, G. (2012b) Pragmatism vs. Interpretivism in Qualitative Information Systems Research, European Journal of Information Systems 21 (2): 135-146.

Grajales, F. J., Sheps, S., Ho, K., Novak-Lauscher, H. and Eysenbach, G. (2014) Social Media: A Review and Tutorial of Applications in Medicine and Health Care, Journal of Medical Internet Research 16 (2): e13.

Gregor, S. and Hevner, A. R. (2013) Positioning and Presenting Design Science Research for Maximum Impact, MIS Quarterly 37 (2): 337-356.

Grisot, M. and Vassilakopoulou, P. (2013) Infrastructures in Healthcare: The Interplay between Generativity and Standardization, International Journal of Medical Informatics 82 (5): e170-e179.

Gross, R. and Acquisti, A. (2005) Information Revelation and Privacy in Online Social Networks, in Proceedings of the 2005 ACM Workshop on Privacy in the Electronic Society (Alexandria, VA, 2005), pp. 71-80.

Haddad, P., Gregory, M. and Wickramasinghe, N. (2014) Business Value of IT in Healthcare, in Wickramasinghe, N., Al-Hakim, L., Gonzalez, C. and Tan, J. (eds.) Lean Thinking for Healthcare: Springer New York, pp. 55-81.

Harsu, M. (2002) A Survey on Domain Engineering, Tampere, Finland: Institute of Software Systems, Tampere University of Technology.

Hevner, A. R., March, S. T., Park, J. and Ram, S. (2004) Design Science in Information Systems Research, MIS Quarterly 28 (1): 75-105.

Hirschfeld, L. A. and Gelman, S. A. (1994) Toward a Topography of Mind: An Introduction to Domain Specificity Mapping the Mind, in Hirschfeld, L. A. and Gelman., S. A. (eds.) Mapping the Mind - Domain Specificity in Cognition and Culture, Cambridge: Cambridge University Press, pp. 3-36.

Hirschheim, R. and Newman, M. (1991) Symbolism and Information Systems Development: Myth, Metaphor and Magic, Information Systems Research 2 (1): 29-62. Holmström, J., Ketokivi, M. and Hameri, A.-P. (2009) Bridging Practice and Theory: A Design Science Approach, Decision Sciences 40 (1): 65-87. 
Iivari, J. (2007) A Paradigmatic Analysis of Information Systems as a Design Science, Scandinavian Journal of Information Systems 19 (2): 39-63.

Iivari, J. (2015) Distinguishing and Contrasting Two Strategies for Design Science Research, European Journal of Information Systems 24 (1): 107-115.

Ives, B. and Olson, M. (1984) User Involvement and MIS Success: A Review of Research, Management Science 30 (5): 586-603.

Jain, S. H. (2009) Practicing Medicine in the Age of Facebook, The New England Journal of Medicine 361 (7): 649-651.

Jones, N. and Thomas, P. (2007) Inter-Organizational Collaboration and Partnerships in Health and Social Care: The Role of Social Software, Public Policy and Administration 22 (3): 289-302.

Kamel Boulos, M. N. and Wheeler, S. (2007) The Emerging Web 2.0 Social Software: An Enabling Suite of Sociable Technologies in Health and Health Care Education, Health Information \& Libraries Journal 24 (1): 2-23.

Kang, K., Kim, S., Lee, J., Kim, K., Shin, E. and Huh, M. (1998) Form: A FeatureOriented Reuse Method with Domain-Specific Reference Architectures, Annals of Software Engineering 5 (1): 143-168.

Kietzmann, J.H., Hermkens, K., McCarthy, I. P. and Silvestre, B.S. (2011) Social Media? Get Serious! Understanding the Functional Building Blocks of Social Media, Business Horizons 54(3): 241-251.

King, S., Greidanus, E., Carbonaro M., Drummond, J. and Patterson, S. (2009) Merging Social Networking Environments and Formal Learning Environments to Support and Facilitate Interprofessional Instruction, Medical Education Online 14(5): 1-9.

Kvarnström, S. (2008) Difficulties in Collaboration: A Critical Incident Study of Interprofessional Healthcare Teamwork, Journal of Interprofessional Care 22 (2): 191203.

Lefebvre, R. C. and Bornkessel, A. S. (2013) Digital Social Networks and Health, Circulation 127 (17): 1829-1836.

Leonardi, P. M. (2014) Social Media, Knowledge Sharing, and Innovation: Toward a Theory of Communication Visibility, Information Systems Research 25 (4): 796-816.

Lieberman, H., and Selker, T. (2000) Out of Context: Computer Systems That Adapt to, and Learn from Context, IBM Systems Journal, 39(3.4): 617-632. 
Lipman, T. (2000) Power and Influence in Clinical Effectiveness and Evidence-Based Medicine, Family Practice 17 (6): 557-563.

Luo, J. S. and Smith, B. N. (2011) Social Networking, Health 2.0, and Beyond, in Dewan, N., Luo, J. S. and Lorenzi, N. M. (eds.) Information Technology Essentials for Behavioral Health Clinicians, London: Springer, pp. 119-131.

Mansfield, S. J., Morrison, S. G., Stephens, H. O., Bonning, M. A., Wang, S.-H. W., J., A. H., Olver, R. C. and Perry, A. W. (2011) Social Media and the Medical Profession, The Medical Journal of Australia 194 (12): 642-644.

March, S. T. and Smith, G. G. (1995) Design and Natural Science Research on Information Technology, Decision Support Systems 15 (4): 251-266.

Mathiassen, L. and Nielsen, P. A. (2008) Engaged Scholarship in IS Research, Scandinavian Journal of Information Systems 20 (2): 3-20.

McAfee, A. (2009) Enterprise 2.0: New Collaborative Tools for Your Organization's Toughest Challenges, Boston: McGraw-Hill.

Merrick, Z., Joanne, G. and Scott, R. (2009) Interprofessional Collaboration: Effects of Practice-Based Interventions on Professional Practice and Healthcare Outcomes, Cochrane Database of Systematic Reviews 8 (3): CD000072.

Mettler, T. (2013) Explorative Clustering of Clinical User Profiles: A First Step Towards User-Centered Health Information Systems, in Proceedings of the 21st European Conference on Information Systems (Utrecht, The Netherlands, 2013), pp. 1-11.

Miller, M., Marks, A. and DeCoulode, M. (2011) Social Software for Business Performance. The Missing Link in Social Software: Measurable Business Performance Improvements, New York: Deloitte.

Musiał, K. and Kazienko, P. (2013) Social Networks on the Internet, World Wide Web 16 (1): 31-72.

Peffers, K., Tuunanen, T., Rothenberger, M. A. and Chatterjee, S. (2008) A Design Science Research Methodology for Information Systems Research, Journal of Management Information Systems 24 (3): 45-77.

Pries-Heje, J. and Baskerville, R. (2008) The Design Theory Nexus, MIS Quarterly 32 (4): 731-755.

Reagans, R., Zuckerman, E. and McEvilly, B. (2004) How to Make the Team: Social Networks vs. Demography as Criteria for Designing Effective Teams, Administrative Science Quarterly 19 (1): 101-133. 
Reinhartz-Berger, I., Sturm, A., Clark, T., Cohen, S. and Bettin, J. (2013). Domain Engineering. Product Lines, Languages, and Conceptual Models, Berlin: Springer.

Reiser, S. J. (1981) Medicine and the Reign of Technology, New York: Cambridge University Press.

Rittel, H. W. J. and Webber, M. M. (1973) Dilemmas in a General Theory of Planning, Policy Sciences 4 (2): 155-169.

Sandars, J. and Schroter S. (2007) Web 2.0 Technologies for Undergraduate and Postgraduate Medical Education: An Online Survey. Postgraduate Medical Journal 83: 759-762.

San Martín-Rodríguez, L., Beaulieu, M.-D., D'Amour, D. and Ferrada-Videla, M. (2005) The Determinants of Successful Collaboration: A Review of Theoretical and Empirical Studies, Journal of Interprofessional Care 19 132-147.

Santos de Oliveira, L. and Gerosa, M. (2011) Collaborative Features in Content Sharing Web 2.0 Social Networks: A Domain Engineering Based on the 3c Collaboration Model, in Vivacqua, A., Gutwin, C. and Borges, M. S. (eds.) Collaboration and Technology, Berlin, Heidelberg: Springer, pp. 142-157.

Scherer, S. A. (2014) Advocating for Action Design Research on IT Value Creation in Healthcare, Journal of the Association for Information Systems 15 (12): 860-878.

Schmid, K. (2000) Scoping Software Product Lines, in Donohoe, P. (ed.) Software Product Lines, Experience and Research Directions, Norwell, MA: Kluwer Academic Publisher, pp. 513-532.

Sein, M. K., Henfridsson, O., Purao, S., Rossi, M. and Lindgren, R. (2011) Action Design Research, MIS Quarterly 35 (1): 37-56.

Sherif, K., Hoffman, J. and Thomas, B. (2006) Can Technology Build Organizational Social Capital? The Case of a Global IT Consulting Firm, Information \& Management 43 (7): 795-804.

Silic, M. and Back, A. (2014) Shadow IT - A View from Behind the Curtain, Computers and Security, 45: 274-283.

Simon, H. A. (1996) The Sciences of the Artificial, Cambridge, MA: MIT Press.

Sniderman, A. D., LaChapelle, K. J., Rachon, N. A. and Furberg, C. D. (2013) The Necessity for Clinical Reasoning in the Era of Evidence-Based Medicine, Mayo Clinic Proceedings 88 (10): 1108-1114. 
Spagnoletti, P., Resca, A. and Saebo, Ø. (2015) Design for Social Media Engagement: Insights from Elderly Care Assistance, Journal of Strategic Information Systems 24 (2): $128-145$.

Stocker, A., Richter, A., Hoefler, P. and Tochtermann, K. (2012) Exploring Appropriation of Enterprise Wikis, Computer Supported Cooperative Work 21 (2-3): 317-356.

Stutzman, F. and Hartzog, W. (2012) Boundary Regulation in Social Media, in Proceedings of the ACM 2012 Conference on Computer Supported Cooperative Work (Seattle, WA, 2012), pp. 769-778.

Susman, G. (1983) Action Research: A Sociotechnical Perspective, in Morgan, G. (ed.) Beyond Method: Strategies for Social Research, Newbury Park, CA: Sage Publications, pp. 95-113.

Swiss Federal Statistical Office (2014) Hospital Statistics, Neuchâtel, Switzerland: Swiss Federal Statistical Office.

Thackeray, R., Neiger, B. L., Smith, A. K. and Van Wagenen, S. B. (2012) Adoption and Use of Social Media among Public Health Departments, BMC Public Health 12 (242): $1-6$.

Thompson, L. A., Black, E., Duff, W. P., Paradise Black, N., Saliba, H. and Dawson, K. (2011) Protected Health Information on Social Networking Sites: Ethical and Legal Considerations, Journal of Medical Internet Research 13 (1): e8.

Tracz, W., Coglianese, L. and Young, P. (1993) A Domain-Specific Software Architecture Engineering Process Outline, SIGSOFT Software Engineering Notes 18 (2): 40-49.

Treem, J. W. and Leonardi, P. M. (2013) Social Media Use in Organizations: Exploring the Affordances of Visibility, Editability, Persistence, and Association, in Salmon, C. T. (ed.) Communication Yearbook, New York: Routledge, pp. 143-189.

Tunnecliff, J., Ilic, D., Morgan, P., Keating, J., Gaida, E. J., Clearihan, L., Sadasivan, S., Davies, D., Ganesh, S., Mohanty, P., Weiner, J., Reynolds, J. and Maloney, S. (2015) The Acceptability among Health Researchers and Clinicians of Social Media to Translate Research Evidence to Clinical Practice: Mixed-Methods Survey and Interview Study, Journal of Medical Internet Research 17 (5): e119.

Vaishnavi, V. and Kuechler, W. (2008) Design Science Research Methods and Patterns: Innovating Information and Communication Technology, Boston, MA: Auerbach Publications. 
Van Aken, J. E. (2005) Management Research as a Design Science: Articulating the Research Products of Mode 2 Knowledge Production in Management, British Journal of Management 16 (1): 19-36.

Venable, J. (2006) The Role of Theory and Theorising in Design Science Research, in Proceedings of the 1st International Conference on Design Science Research in IT (Claremont, CA, 2006), pp. 1-18.

Walls, J. H., Widmeyer, G. R. and El Sawy, O. A. (1992) Building an Information Systems Design Theory for Vigilant Eis, Information Systems Research 3 (1): 36-59.

Walsh, J. P. and Maloney, N. G. (2007) Collaboration Structure, Communication Media, and Problems in Scientific Work Teams, Journal of Computer-Mediated Communication 12 (2): 712-732.

Wasko, M. M. and Faraj, S. (2005) Why Should I Share? Examining Social Capital and Knowledge Contribution in Electronic Networks of Practice, MIS Quarterly 29 (1): 3557.

Wellman, B. and Berkowitz, S. D. (1988) Social Structures: A Network Approach, New York: Cambridge University Press.

White, J., Kirwan, P., Lai, K., Walton, J. and Ross, S. (2013) 'Have You Seen What Is on Facebook?' the Use of Social Networking Software by Healthcare Professions Students, BMJ Open 3 (7): e003013.

Wiener, L., Crum, C., Grady, C. and Merchant, M. (2012) To Friend or Not to Friend: The Use of Social Media in Clinical Oncology, Journal of Oncology Practice 8 (2): 103106.

Yamashita, N., Inaba, R., Kuzuoka, H. and Ishida, T. (2009) Difficulties in Establishing Common Ground in Multiparty Groups Using Machine Translation, in Proceedings of the SIGCHI Conference on Human Factors in Computing Systems (Boston, MA, 2009), pp. 679-688.

Zhang, J., Qu, Y., Cody, J. and Wu, Y. (2010) A Case Study of Micro-Blogging in the Enterprise: Use, Value, and Related Issues, in Proceedings of the SIGCHI Conference on Human Factors in Computing Systems (New York, NY, 2010), pp. 123-132.

Zhao, D. and Rosson, M. B. (2009) How and Why People Twitter: The Role That MicroBlogging Plays in Informal Communication at Work, in Proceedings of the ACM 2009 international conference on Supporting group work (Sanibel Island, FL, 2009), pp. 243-252. 
Zheleva, E. and Getoor, L. (2009) To Join or Not to Join: The Illusion of Privacy in Social Networks with Mixed Public and Private User Profiles, in Proceedings of the 18th International Conference on World Wide Web (Madrid, Spain, 2009), pp. 531-540. 


\section{APPENDIX A - TECHNICAL IMPLEMENTATION OF healthcare connect}

In this appendix, we describe the technical implementation of our healthcare connect PSN. We used the open source software Liferay as starting point for building our solution. Liferay is written in Java and provides all necessary features, which are required to develop and maintain an enterprise portal. Liferay makes use of so-called portlets, which allows it to easily configure a PSN. Figure A1 illustrates the technical architecture and the most important server-side portlets we used in our PSN. Special features, such as our benchmarking component, were integrated as web content. Our PSN was designed for both, desktop PC and mobile devices. We also set up a secure network environment. The prototype was iteratively tested and refined in order to ensure suitability and performance of the solution. At this stage, the prototype is being augmented to reach marketability.

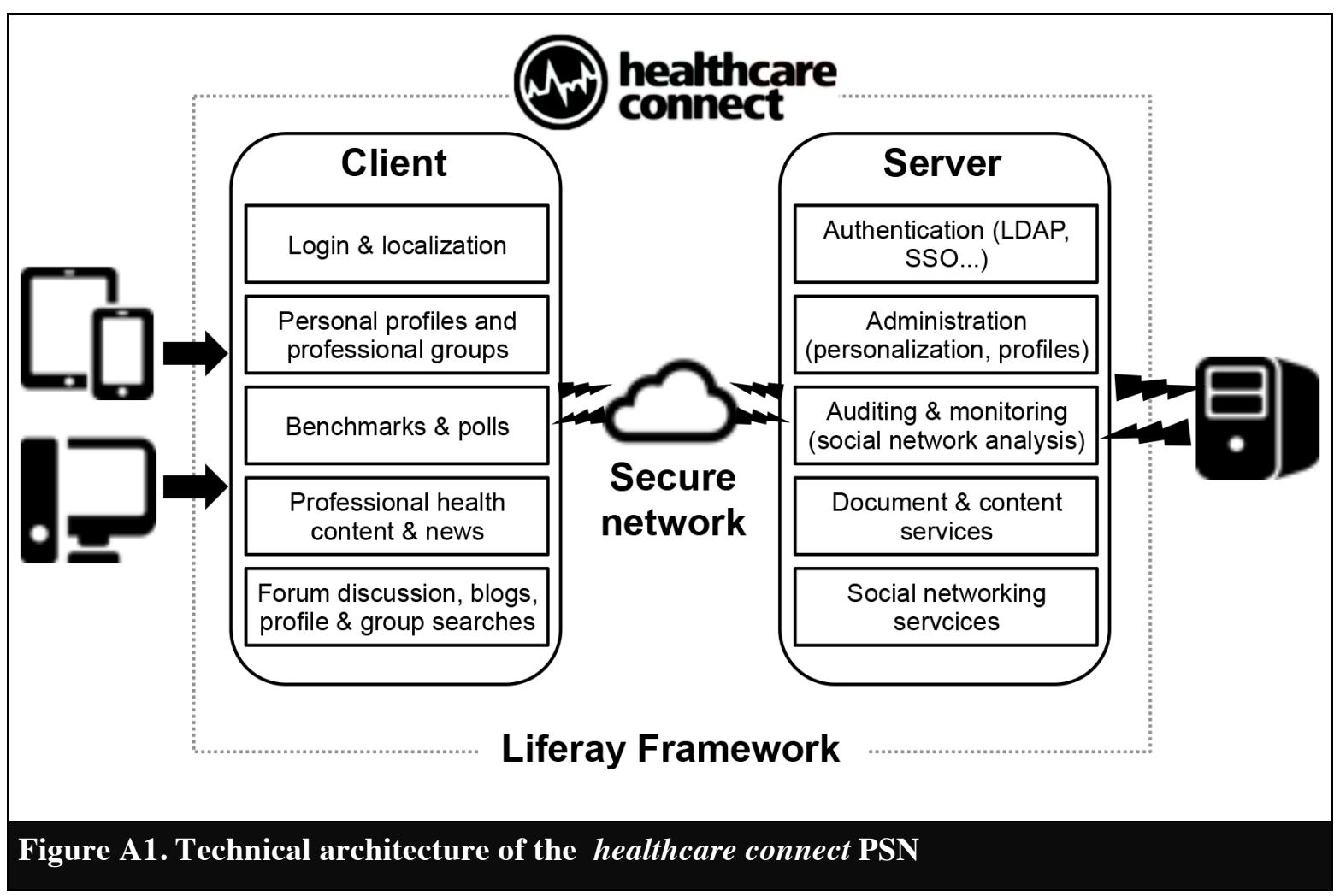




\section{APPENDIX B - ITERATIVE ADAPTATION OF healthcare connect}

Following the ADR approach, we applied an iterative approach of dedicated building, intervention, and evaluation activities before reaching to the version of the PSN discussed in this article. At first, we used paper-based descriptions and mockups to translate the verbalized requirements of the interviews and focus group sessions into a visual design. Based on this, an early software prototype was developed in order to explore the ADR team's design hypotheses. The reactions to the first software-based prototype were helpful for adapting the solution to its final design that had a particular emphasis on the inclusion of domain-specific content. Figure B1 shows an example of a major design shift from the first prototype to the final design of the PSN.

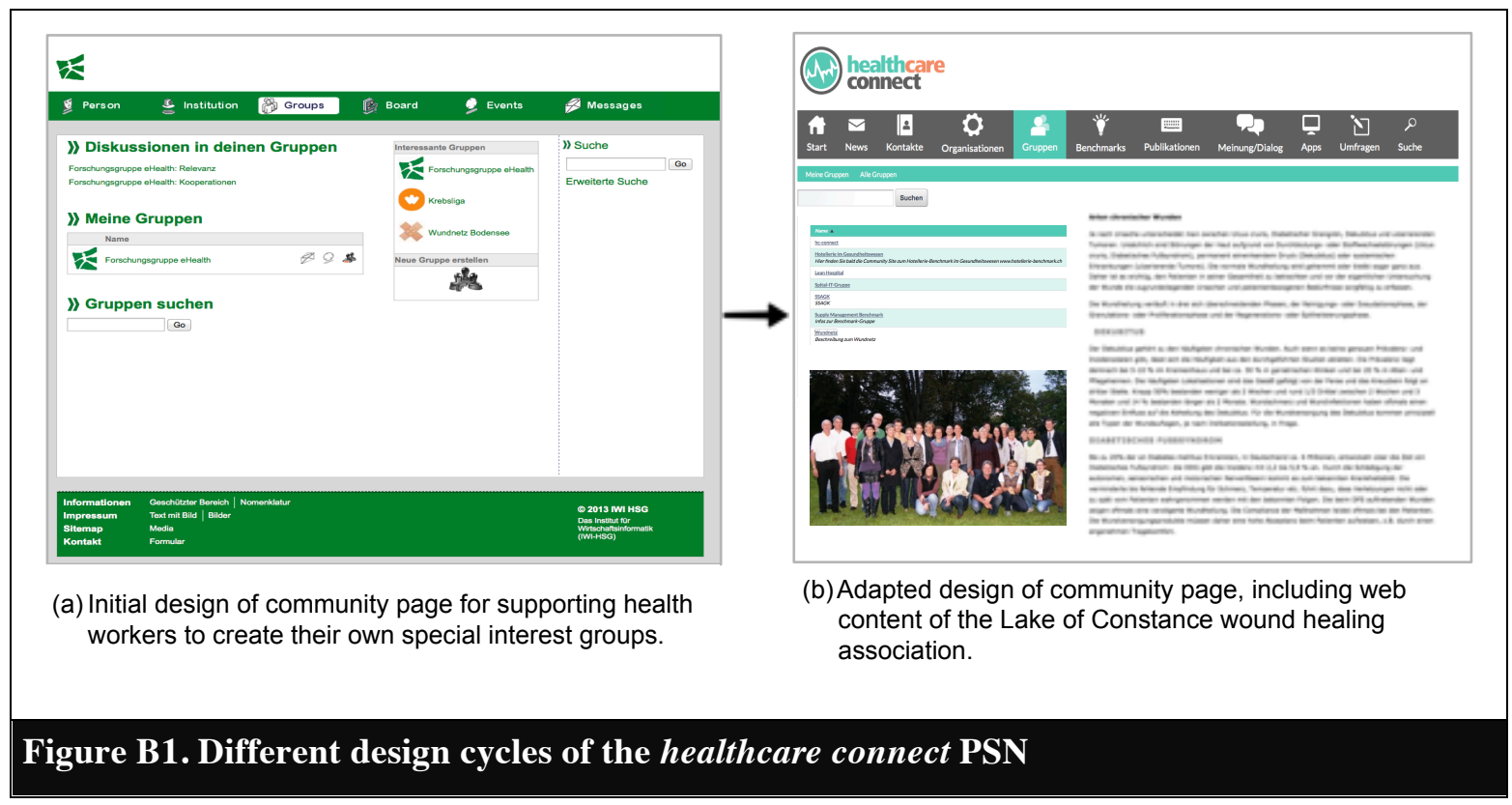

